drugs. If the co-operation, therefore, between physician and surgeon is indispensable to the patient's welfare, it has become equally indispensable to the preparation of a modern manual.

These two volumes comprise a collection of essays contributed by experts in their particular subject. It was not designed as a textbook; yet.it has become if not a textbook in name, certainly a work of reference in fact. A vast deal of ground has been covered with remarkably little overlap considering the number of individual contributors.

The first volume is concerned chiefly with the general aspects of the subject, such as epidemiology, the clinical and laboratory diagnosis of the disease, morbid anatomy, radiology, administration and rehabilitation. The minimal lesion with its special problems, fluorography, B.C.G. inoculation, psychology and tuberculosis in industry are all discussed. The second volume deals with the disease more precisely by systems and contains accounts of the surgical methods employed in treatment.

Considering the entire work it can be said that the main editorial difficulty of blending so large a number of separate contributions into a composite whole has been overcome. The result is a manual that is authoritative and complete, and one which will enhance the prestige of British medical practice.

\section{A SHORT PRACTICE OF SURGERY}

ByHamilton Bailey, F.R.C.S., F.A.C.S., F.I.C.S., F.R.S.E., and R. J. McNeILl Love, M.S., F.R.C.S., F.A.C.S., F.I.C.S. 9th Edition. Pp. viii + I,254, with 1,234 illustrations. London: H. K. Lewis \& Co., Ltd. 1952.

This excellent book is now presented in its ninth edition. The authors regret that owing to surgical advances they have had to enlarge it slightly. There are 206 extra pages and it is now as large as it can be if it is to be retained as a single volume work. It has been extensively revised and brought up to date. Portal hypertension has a section to itself and the peptic ulcer chapter has been enlarged. A more comprehensive account of carcinomata of the pharynx and larynx is welcomed. In the chapter on arterial disease there is a detailed account of modern methods of treatment but it is a pity that there has been no mention of arteriography in diagnosis. The illustrations, many of which have been changed, are excellent.

This book will justly continue to be one of the most popular books for medical students.

$$
\text { D.L.B.F. }
$$

\section{INTRODUCTION TO CLINICAL NEUROLOGY}

By Gordon Holmes, M.D., F.R.S. 2nd Edition. Pp. vii + I 89, with 43 illustrations. Edinburgh: E. \& S. Livingstone. I952. I2s. 6d.

This book, as the author writes in his preface, does not "describe or deal with injuries and diseases as such,' but it discusses 'the nature and the significance of the symptoms and abnormal signs which a patient with a nervous disorder may present or which may be revealed by clinical examina.'

It is a most excellent introduction to this aspect of the study of neurology. It is also more than an introduction as it is valuable and refreshing to the initiated. Whatever its title may suggest it is emphatically a book for practitioners as well as students.

To some extent it treats in cross section the facts which are listed, so to speak, longitudinally in text books of neurology, and in so doing it elucidates and compares the same or similar signs and symptoms in different diseases.

Here are some of its chapter headings: The Motor Systems, Muscle Tone and Co-ordination of Movement, Examination of the Motor System, Sensation, The Examination of Sensation, Reflexes, The Visual System, Speech and its Disorders, Agnosia and Apraxia, The Bladder and Rectum.

This somewhat random selection gives some idea of the plan. A book with this approach is refreshing and intriguing to read, especially one as thoughtful, compact and full of experience as this. There are all too few of its kind.

To the reviewer's knowledge there is certainly no book in the British literature which covers this book's territory in its particular way.

When one remembers the striking clarity of the author's bedside demonstrations it is surprising to find that the book contains a number of obscure sentences. Some are so because they are cumbersome and others because of inadequate proofreading. However, they do not seriously detract from an excellent book.

Every aspiring neurologist or medical consultant should read it, as should any student who aims at a competent grasp of the subject of neurology.

$$
\text { C.E. }
$$

\section{THE RHESUS FACTOR}

By G. Fulton Roberts, M.A., M.D. 3rd Edition.

Pp. vii +90 . London: William Heinemann. I952. 5s.

This booklet gives an excellent account of the Rhesus factor and its clinical implications. All the relevant information is clearly set out, and many case reports further illustrate the various points raised in the text. Naturally, the main appeal will be to those engaged in blood transfusion and obstetric practice, but the importance of the subject and clarity of presentation will make this booklet highly acceptable to all practitioners.

M.H.

\section{TUBERCULOSIS OF BONE AND JOINT}

By The Late G. R. Girdlestone and E. W. Somerville, M.B., F.R.C.S. 2nd Edition. Pp. xii +314 , with 182 illustrations. London: Geoffrey Cumberlege. 1952. 45s.

Published after the untimely death of Professor Girdlestone, this second edition will keep fresh the memory of a brilliant surgeon with the widest type 